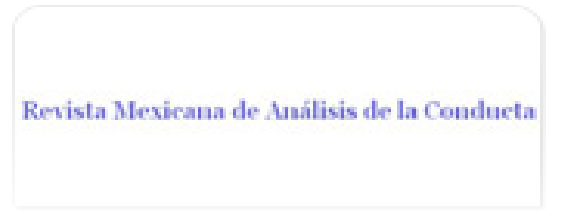

Revista Mexicana de Análisis de la Conducta ISSN: 0185-4534

editora@rmac-mx.org

Sociedad Mexicana de Análisis de la Conducta México

Prado-Alcalá, Roberto A.; Cobos-Zapiaín, Guillermo; Salado-Castillo, Rigoberto; Quiroz, César; GarínAguilar, María Eugenia; Díaz, Arnulfo; Díaz Del Guante, Miguel Ángel; Medina, Andrea C.; Martínez, Isabel; Quirarte, Gina L.

El aprendizaje incrementado protege a la memoria contra tratamientos amnésicos

Revista Mexicana de Análisis de la Conducta, vol. 32, núm. 2, diciembre, 2006, pp. 203-218

Sociedad Mexicana de Análisis de la Conducta

Guadalajara, México

Disponible en: http://www.redalyc.org/articulo.oa?id=59332208

Cómo citar el artículo

- Número completo

- Más información del artículo

Página de la revista en redalyc.org

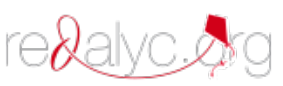

Sistema de Información Científica

Red de Revistas Científicas de América Latina, el Caribe, España y Portugal Proyecto académico sin fines de lucro, desarrollado bajo la iniciativa de acceso abierto 


\title{
EL APRENDIZAJE INCREMENTADO PROTEGE A LA MEMORIA CONTRA TRATAMIENTOS AMNÉSICOS
}

\author{
ENHANCED LEARNING PROTECTS \\ MEMORY AGAINST AMNESIC \\ TREATMENTS
}

\begin{abstract}
ROBERTO A. PRADO-ALCALÁAB, GUILLERMO COBOS-ZAPIAÍNA, RIGOBERTO SALADO-CASTILLO' ${ }^{\circ}$, CÉSAR QUIROZA, MARÍA EUGENIA GARÍN-AGUILAR', ARNULFO DÍAZA, MIGUEL ÁNGEL DÍAZ DEL GUANTED, ANDREA C. MEDINAA , ISABEL MARTÍNEZ ${ }^{\mathrm{E}}$ Y GINA L. QUIRARTEA

AINSTITUTO DE NEUROBIOLOGÍA, DEPARTAMENTO DE NEUROBIOLOGÍA CONDUCTUAL Y COGNITIVA, CAMPUS JURIQUILLAQUERÉTARO Y ${ }^{\mathrm{B}} F E S-I Z T A C A L A$, UNIVERSIDAD NACIONAL AUTÓNOMA DE MÉXICO; 'UNIVERSIDAD DE PANAMÁ; DUNIVERSIDAD VERACRUZANA; EUNIVERSIDAD AUTÓNOMA DE PUEBLA
\end{abstract}

\begin{abstract}
RESUMEN
Se hace una descripción somera de las técnicas clásicas utilizadas en el estudio de la neurobiología de la memoria, y de los resultados experimentales que dieron base a la teoría de la consolidación de la memoria. Asimismo, se describen los experimentos que han dado lugar a una reinterpretación de dicha teoría, aplicable a la formación de la memoria de altos niveles de aprendizaje. Los datos derivados de estudios neurobiológicos en los que se han utilizado diseños que implican aprendizajes mediados por niveles rela-

* Varios de los trabajos descritos en este artículo fueron financiados por PAPIIT-DGAPA-UNAM y por CONACYT

Dirigir toda correspondencia a: Dr. Roberto A. Prado Alcalá, Instituto de Neurobiología, Campus Juriquilla-Querétaro, Apdo. Postal 1-1141, Querétaro, Qro. 76001, Querétaro. Tel. (52442) 238-1047; (5255) 5623-4047 FAX: (52442) 238-1046; (5255) 5623-4046.

E-mail: prado@servidor.unam.mx
\end{abstract}


tivamente bajos de reforzamiento o de número de ensayos o de sesiones de entrenamiento, así como los derivados de situaciones de aprendizaje incrementado pueden se explicados en forma sencilla por dos modelos que hemos propuesto: la del procesamiento en serie y la del procesamiento en paralelo, que se discuten en el texto.

Palabras clave: aprendizaje, memoria, amnesia, sistema nervioso, procesamiento en serie, procesamiento en paralelo, sobre-entrenamiento, sobrereforzamiento.

\begin{abstract}
We present a brief description of the classic techniques used in the neurobiological study of memory, and of the experimental results that gave way to the theory of memory consolidation. We also describe those experiments that have given place to a reinterpretation of that theory, applicable to the formation of memory of high levels of learning. The data derived from neurobiological studies of memory, where learning is achieved through relatively low levels of reinforcement, low number of trials, or small number of training sessions, as well as those derived from situations of increased levels of learning, can be explained in a simple manner by two models that we have proposed: serial and parallel processing of information, which are discussed in the text.

Key words: learning, memory, amnesia, nervous system, serial processing, parallel processing, overtraining, overreinforcement.

El inicio del siglo $X X$ coincidió con un avance conceptual que ha guiado la mayor parte de la investigación experimental acerca de la neurobiología de la memoria: la hipótesis de la consolidación de la memoria. Georg Elias Müller y su alumno Alfons Pilzecker publicaron una extensa monografía en la que reportaron 40 experimentos realizados entre 1892 y 1900, para identificar las leyes que rigen la formación y evocación de la memoria. Concibieron el concepto de consolidación de la memoria y lo introdujeron a la literatura científica. Entre las conclusiones más importantes de su trabajo, Müller y Pilzecker (1900) concluyeron que la fijación de la memoria requiere de tiempo (consolidación) y que la memoria es vulnerable durante el período de consolidación.

Los cien años que siguieron a las aportaciones de Müller y Pilzecker han sido testigos de un rápido desarrollo del campo de la neurobiología de la memoria (McGaugh, 2000). Con la ayuda de técnicas propias de la fisiología y la farmacología, asociadas a metodologías neuroanatómicas, se hicieron las primeras descripciones de estructuras cerebrales necesarias para el almacenamiento de información. Así, se encontró que las lesiones permanentes,
\end{abstract}


la estimulación eléctrica o la inactivación reversible de múltiples regiones cerebrales producen deficiencias significativas en la memoria. Posteriormente, administrando sistémicamente (es decir, por vía oral, intraperitoneal, subcutánea o intravenosa) o directamente en el parénquima cerebral fármacos que activan o bloquean la acción de moléculas neurotransmisoras, se describió que algunos sistemas neuroquímicos específicos mediaban la consolidación de la memoria (Bermúdez-Rattoni \& Prado-Alcalá, 2001).

En épocas recientes, con el desarrollo de la biología celular y molecular, se han descrito algunos de los mecanismos íntimos neuronales que son desencadenados a partir de experiencias de aprendizaje, que a través del proceso de consolidación, tienen como consecuencia la formación de la memoria de largo plazo. Así, se sabe que la formación de esta memoria depende de la activación de genes específicos que contienen la codificación necesaria para la síntesis de proteínas. De esta manera, la información derivada de una experiencia de aprendizaje podría quedar almacenada en virtud de cambios en la estructura y metabolismo neuronales (crecimiento dendrítico, génesis de espinas dendríticas, incremento en la producción y liberación de neurotransmisores, síntesis de receptores de membrana sensibles a neurotransmisores específicos, etc.) (Bailey, Bartsch \& Kandel, 1996; Decker \& McGaugh, 1991; Stork \& Welzl, 1999).

En términos generales, los datos obtenidos con el uso de las diferentes metodologías descritas son congruentes entre sí. Es decir, si la lesión de una estructura cerebral especifica produce una memoria disminuida, entonces la interferencia funcional producida por estimulación eléctrica o el bloqueo farmacológico de alguno de sus sistemas de neurotransmisión tienen una consecuencia similar. Por otra parte, la administración local de agentes precursores o agonistas del sistema de neurotransmisión correspondiente puede inducir una mejor memoria.

De esta manera, se han descrito estructuras específicas, cuya actividad es indispensable para que se almacenen diferentes tipos de memoria. Un importante estudio en este sentido, que sirve para ejemplificar la existencia de sistemas múltiples de memoria es el de McDonald y White (1993). En una elegante serie experimental, en la que utilizaron tres versiones del laberinto radial de ocho brazos, demostraron que el daño producido en el estriado impide el establecimiento de la memoria de procedimiento (aprendizaje de hábitos), mientras que las lesiones de la amígdala o del hipocampo no interfieren con ella. Por otra parte, la lesión de la amígdala deteriora la memoria emocional, no así la lesión del estriado o del hipocampo. Del mismo modo, la lesión del hipocampo interfiere con el establecimiento de la memoria espacial, cosa que no ocurre al lesionar el estriado o la amígdala. En otras palabras, encontraron estructuras cerebrales particulares de las que dependen memorias particulares. 
Una tarea de aprendizaje que ha sido ampliamente usada para estudiar procesos de memoria es la de evitación inhibitoria, también denominada prevención pasiva ${ }^{1}$. En este aprendizaje están implicados los tipos de memoria estudiados por McDonald y White: a) tiene un alto componente emocional, ya que se establece mediante la aplicación de un evento aversivo: un choque eléctrico aplicado en las patas de los animales de experimentación, b) se establece mediante claves espaciales, y c) implica la asociación de estímulos ambientales con la respuesta motora. De acuerdo con la propuesta de McDonald y White (1993), el estriado, la amígdala y el hipocampo deberían ser necesarios para el establecimiento de la memoria de esta tarea. De hecho, existe una vasta literatura en la que se demuestra que esta suposición es correcta (e.g., Ambrogi Lorenzini et al., 2000; Bammer, 1982; Izquierdo \& Medina, 1993; McGaugh, Mclntyre \& Power, 2002; Packard \& Knowlton, 2002; Prado-Alcalá et al., 2004)

A pesar de los antecedentes descritos, una serie de estudios experimentales, iniciados en forma sistemática en nuestro laboratorio, indican que la teoría prevaleciente acerca de la consolidación de la memoria, revisada en párrafos anteriores, sólo se puede aplicar a la memoria de aprendizajes mediados por estímulos relativamente débiles, o establecidos a través de un número relativamente bajo de ensayos o de sesiones de entrenamiento. Recuérdese que tal teoría postula que la fijación de la memoria requiere de tiempo (consolidación) y que la memoria es frágil durante este período de consolidación (McGaugh, 2000; Müller \& Pilzecker, 1900).

A continuación haremos un recuento de los datos que nos han llevado a un planteamiento alternativo acerca de cómo se consolida la memoria en situaciones de aprendizajes incrementados. La siguiente reseña no necesariamente seguirá un orden cronológico, sino será ordenada en función del tipo de tratamiento que se administró y de la tarea de aprendizaje que se estudió.

Uno de los sistemas de neurotransmisión que ha recibido más atención por su posible participación en procesos de memoria, es el sistema colinérgico. Aunque con algunas excepciones, numerosos estudios demuestran que drogas antagonistas de la acetilcolina, administradas por vía sistémica o directamente en estructuras cerebrales, producen deterioros significativos en la retención de una gran variedad de conductas aprendidas, incluyendo la de evitación inhibi-

1. En una situación típica de evitación inhibitoria, una rata es colocada en un compartimiento iluminado, separado por una puerta deslizable de otro oscuro. Cuando la puerta es abierta, la rata cruza al compartimiento oscuro (debido a su fotofobia innata). Una vez que ha cruzado, se le administra un leve choque eléctrico en las patas. Veinticuatro horas después se repite el procedimiento y se puede observar que la rata no pasa de nuevo al compartimiento en el que se le aplicó el choque el día anterior. Esta conducta de inhibición implica que la rata almacenó en la memoria de largo plazo la experiencia aversiva. En contraste, las ratas que nunca recibieron el choque durante la primera sesión, vuelven a pasar al compartimiento oscuro al día siguiente. 
toria; asimismo, la administración de agonistas o precursores de la acetilcolina facilitan la formación de la memoria (e.g., Bammer, 1982; Bartus, Dean, Pontecorvo \& Flicker, 1985; Fibiger, 1991; Gold, 2003; Prado-Alcalá, 1985).

De igual forma, la depleción de serotonina cerebral, el bloqueo de receptores a serotonina, o la lesión de las vías serotoninérgicas impiden el desarrollo normal del aprendizaje y la memoria, mientras que la activación de algunos de los 14 subtipos de receptores a serotonina producen una mejoría en estos procesos mnemónicos (Altman \& Normile, 1986; Archer, 1982; Meneses, Manuel-Apolinar, Rocha, Castillo \& Castillo, 2004; Meneses, 1999, 2003; Ogren, 1985, 1986a, 1986b).

Los experimentos, descritos a continuación, reseñan estudios en los que se utilizó la tarea de evitación inhibitoria, a menos que se indique lo contrario.

En 1990, Durán-Arévalo, Cruz-Morales y Prado-Alcalá reportaron que la aplicación intraperitoneal de escopolamina (bloqueador de los receptores muscarínicos a la acetilcolina), a ratas, inmediatamente después del entrenamiento, produjo el efecto amnésico esperado, muchas veces reportado por otros autores. Sin embargo, cuando otros grupos de animales fueron sometidos al mismo entrenamiento, pero utilizando estímulos aversivos del doble o triple de la intensidad que se aplicó cuando se produjo amnesia, no se encontró deterioro alguno en la memoria.

Siguiendo esta línea experimental, Cruz-Morales et al. (1992), realizaron un estudio para determinar si el efecto protector inducido por el incremento en la experiencia de aprendizaje era inducido en forma gradual, o si se tenía que alcanzar cierto umbral de activación para que se diera ese efecto. Entrenaron ratas aplicando, en grupos independientes, intensidades de choque eléctrico que se fueron incrementando en pasos de $0.1 \mathrm{~mA}$. Encontraron que, a pesar de que todas las intensidades produjeron un aprendizaje óptimo en los grupos controles, dentro de un rango de intensidades relativamente bajas, la administración intraperitoneal de escopolamina produjo un fuerte cuadro amnésico, pero a partir de cierta intensidad, bastó con incrementarla en $0.1 \mathrm{~mA}$ (menos del $4 \%$ ), para que a partir de ese punto el tratamiento farmacológico ya no tuviera efecto alguno sobre la memoria.

Posteriormente, se estudiaron los efectos de la escopolamina, también inyectada sistémicamente, sobre la consolidación de la memoria de grupos de ratas entrenadas con choques que, en los grupos controles, indujeron niveles bajos, intermedios o altos de aprendizaje. Como era de esperarse, la escopolamina produjo el típico cuadro amnésico en los grupos con un nivel de entrenamiento intermedio, y ningún efecto en los animales con un alto nivel de entrenamiento (choque de mayor intensidad). Sorpresivamente, los animales con un bajo nivel de entrenamiento (choque de baja intensidad) no mostraron deficiencia alguna en su memoria (Quirarte, Cruz-Morales, Díaz del Guante, García \& Prado-Alcalá, 1993). 
En conjunto, estos datos indican que la actividad colinérgica cerebral es indispensable para que se lleve a cabo la consolidación de la memoria cuando hay un nivel "intermedio" de aprendizaje, es decir, cuando la magnitud de los estímulos reforzantes no es muy grande ni muy pequeña. También puede decirse que el aprendizaje incrementado protege a la memoria en contra de tratamientos amnésicos de agentes anticolinérgicos.

Una pregunta cardinal fue: ¿se pueden generalizar estos resultados a otros sistemas neuroquímicos?

En una primera aproximación, la respuesta fue negativa. Solana-Figueroa, Quirarte y Prado-Alcalá (1999) utilizaron p-cloroanfetamina (PCA); al administrarse intraperitonealmente, este fármaco produce una importante depleción de serotonina cerebral, ya que causa la lesión de neuronas que sintetizan y axones que contienen este neurotransmisor. Ratas tratadas de esta manera fueron entrenadas en la tarea de evitación inhibitoria, aplicándoseles una intensidad de choque relativamente baja u otra relativamente alta. Veinticuatro horas después se probó su memoria. La PCA indujo un cuadro amnésico, independientemente de la intensidad del estímulo aversivo.

Sin embargo, en un estudio posterior, Solana-Figueroa et al. (2002) aplicaron el mismo tratamiento a ratas que fueron entrenadas con choques eléctricos de intensidades que abarcaban a las del primer estudio, así como intensidades mayores. En esta nueva situación, la PCA produjo la amnesia esperada en los animales entrenados con las intensidades bajas, pero no hubo efecto alguno en los grupos entrenados con las intensidades más altas, confirmándose el efecto protector del aprendizaje incrementado, ahora en contra de los efectos amnésicos de una droga que interfiere con la actividad serotoninérgica cerebral.

Los datos descritos indican claramente que el aprendizaje incrementado impide que se produzcan estados amnésicos que típicamente se observan como consecuencia de la aplicación sistémica de drogas anticolinérgicas y antiserotoninérgicas. Sin embargo, los experimentos descritos no permiten saber en cuales zonas cerebrales se están llevando a cabo los efectos de los tratamientos aplicados. Para saberlo, se diseñaron experimentos en los que se administraron fármacos en regiones discretas del cerebro.

Giordano y Prado-Alcalá (1986) describieron que el bloqueo colinérgico del estriado, inducido por la administración local de atropina unos minutos después del entrenamiento, interfiere con la retención de la evitación inhibitoria. Lo más interesante de este estudio es que ese mismo tratamiento farmacológico no produjo ningún cambio en la memoria en animales que recibieron un estímulo aversivo de intensidad relativamente alta durante el entrenamiento. Unos años más tarde Díaz del Guante, Rivas-Arancibia, Quirarte y Prado-Alcalá (1990) confirmaron este efecto.

Estos resultados obtenidos al someter a los animales a un aprendiza- 
je incrementado (inducido por un alto nivel de reforzador negativo), indicaban fuertemente que el sistema colinérgico estriatal ya no interviene en el proceso de consolidación o de retención de la memoria, dieron lugar a dos hipótesis alternativas para explicar el efecto protector encontrado: a) que la participación del estriado ahora depende de otros sistemas de neurotransmisión del mismo estriado, o b) que el estriado ya no es necesario para que se consolide la memoria. Si la segunda hipótesis resultara ser correcta, la primera sería descartada. Así pues, la segunda hipótesis fue sometida a la prueba experimental, entrenando ratas con intensidades relativamente bajas o altas de choque eléctrico; inmediatamente después del entrenamiento se les microinyectó, en el estriado dorsal, lidocaína ${ }^{2}$, y se probó la memoria 24 horas más tarde. Como era de esperarse, los animales entrenados con una intensidad baja presentaron un cuadro amnésico considerable; por otro lado, los entrenados con una intensidad de choque relativamente alta tuvieron una memoria óptima. Estos resultados indican que en condiciones de alto entrenamiento el estriado, como un todo, deja de ser indispensable para que la memoria se consolide (Pérez-Ruiz y Prado-Alcalá, 1989).

Debido a la importancia del efecto protector encontrado, resolvimos explorar la posibilidad de que éste también se encontrara en otros núcleos cerebrales que participan en procesos mnemónicos. El primero que seleccionamos fue la substantia nigra, ya que está conectada directa y bidireccionalmente con el estriado, además de que es una estructura que indudablemente participa en la formación de la memoria, y porque ambos núcleos forman parte del sistema nigroestriatal, que además de participar en procesos de memoria, también lo hacen en la regulación motora. (e.g., Ambrogi-Lorenzini, Baldi, Bucherelli y Tassoni, 1994; Da Cunha et al., 2002, 2003; Díaz del Guante, Rivas, Prado-Alcalá \& Quirarte, 2004; Routtenberg \& Holzman, 1973).

Una de las principales proyecciones del estriado hacia la substantia nigra es GABAérgica, por lo que Cobos-Zapiaín et al. (1996) decidieron explorar los efectos de la administración de bicuculina y de picrotoxina (bloqueadores de la acción del GABA) en la substantia nigra, en grupos independientes de ratas entrenadas con alto o bajo nivel de estimulación aversiva. Los resultados ya no fueron sorprendentes: ambas drogas produjeron amnesia en los grupos de bajo entrenamiento, mientras que en los entrenados con un alto nivel de choque eléctrico no sufrieron deterioro alguno en su memoria.

Otras dos regiones cerebrales involucradas en el aprendizaje de tipo

2. La lidocaína es un anestésico local, que bloquea los canales de sodio de las membranas axónicas, impidiendo con ello la producción y propagación de potenciales de acción, por lo que impide la comunicación interneuronal y por lo tanto cualquier actividad integrativa. Su efecto es reversible. 
aversivo son la amígdala y el hipocampo, pertenecientes al sistema límbico, también participando, la primera de ellas, en el procesamiento de información emocional o afectiva, y la segunda, en la integración de claves espaciales. Una prueba importante para determinar el grado de generalización del efecto de protección de la memoria producido por aprendizajes incrementados fue estudiar si lo descrito para el sistema nigroestriatal también tenía su contraparte en el sistema límbico. Tal fue el caso, como veremos a continuación.

Muchas evidencias experimentales apoyan la idea de que la amígdala participa en la formación de la memoria (ver revisiones de McGaugh, 2002; Power, 2004; Rodrigues, Schafe \& LeDoux, 2004). En una importante serie de experimentos realizados en el laboratorio de J. L. McGaugh, Parent et al. demostraron que las lesiones permanentes o la inactivación reversible de la amígdala de ratas entrenadas con esquemas de múltiples ensayos o con intensidades relativamente altas de choques eléctricos, al probar su memoria no se produjo la amnesia que típicamente se encuentra en ratas con bajos niveles de entrenamiento (Parent, Tomaz \& McGaugh, 1992; Parent \& McGaugh, 1994; Parent, West \& McGaugh, 1994; Parent, Quirarte, Cahill \& McGaugh,1995).

En congruencia con esos resultados, Salado-Castillo, Sánchez-Alavez, Quirarte y Prado-Alcalá (1995) reportaron que la aplicación de lidocaína en la amígdala, en el estriado o en la substantia nigra, inmediatamente después del entrenamiento produce un profundo estado amnésico en ratas entrenadas con niveles bajos de choque eléctrico, pero no observaron ningún efecto sobre la memoria en ratas entrenadas con un nivel alto del estímulo aversivo.

En experimentos recientes, Martínez et al. (2002) encontraron que la lesión permanente de las áreas CA1 y CA3 del hipocampo, inducida por la administración de ácido kaínico, produce el consabido deterioro en la memoria de largo plazo; sin embargo, si en animales lesionados se prueba la memoria de corto plazo, ésta se encuentra intacta. Con el objeto de determinar si el aprendizaje incrementado contrarresta el efecto amnésico de la interferencia de la actividad del hipocampo, Quiroz et al. (2003) inactivaron reversiblemente esta estructura, microinyectando tetrodotoxina ${ }^{3}$ inmediatamente después del entrenamiento.

Los datos obtenidos de los animales sometidos a un alto nivel de aprendizaje, a los que se les lesionó crónicamente o se les inactivó reversiblemente la amígdala o el hipocampo, indican que el efecto protector descrito puede generalizarse a estructuras pertenecientes al sistema límbico.

3. La tetrodotoxina bloquea los canales de sodio de las membranas axónicas, impidiendo con ello la producción y propagación de potenciales de acción, por lo que impide la comunicación interneuronal y por lo tanto cualquier actividad integrativa. Su efecto es reversible. 
Hasta aquí, los experimentos que hemos descrito, relacionados con la protección de la memoria inducida por alto nivel de entrenamiento, se han referido al aprendizaje y memoria de la tarea de evitación inhibitoria, que representa un aprendizaje mediado por eventos aversivos. Pero, ¿se producirá el efecto protector cuando el aprendizaje sea mediado por eventos gratificantes (reforzadores positivos)? La respuesta a esta pregunta es positiva, como veremos a continuación ${ }^{4}$.

El efecto de la microinyección de atropina en el núcleo caudado ${ }^{5}$ de gatos sobre la retención de una tarea instrumental reforzada positivamente (presionar una palanca para recibir leche) fue descrito por primera vez en la década de 1970 (Prado-Alcalá et al., 1972). En esta misma época, también se describió, por primera vez, que cuando esta tarea instrumental es sobreentrenada, el bloqueo colinérgico del caudado ya no interfiere con la memoria de esa tarea (Prado-Alcalá \& Cobos-Zapiaín, 1977). Posteriormente, PradoAlcalá, Bermúdez-Rattoni, Velázquez-Martínez \& Bacha (1978) encontraron el mismo efecto protector al inyectar un antagonista colinérgico en el estriado de ratas entrenadas en un aprendizaje de alternancia espacial reforzado positivamente.

El siguiente paso lógico fue determinar si era la actividad colinérgica del caudado de gatos o del estriado de ratas la que dejaba de intervenir en la memoria de aprendizajes incrementados, o si, como para el caso de la evitación inhibitoria, esas estructuras, como un todo, dejaban de participar. Para ello, tanto a gatos (Prado-Alcalá \& Cobos-Zapiaín, 1979) como a ratas (PradoAlcalá, Kaufman \& Moscona, 1980) se les entrenó en la tarea instrumental de presionar una palanca durante un número bajo, intermedio o alto de sesiones. Después del entrenamiento se probó su memoria bajo los efectos de la microinyección de una alta concentración de potasio $(K C L 3 M)^{6}$ en las estructuras referidas. Los resultados de estos experimentos fueron equivalentes: los grupos con un bajo número de sesiones presentaron una marcada

4. De hecho, cronológicamente, los primeros estudios sistemáticos al respecto se hicieron utilizando reforzadores positivos. En esta presentación, en aras de una mayor claridad de la exposición, hemos hecho un recuento guiado por las metodologías empleadas para la administración de los tratamientos, así como por el tipo de aprendizaje utilizado.

5. El núcleo caudado forma parte de los ganglios basales, y recibe este nombre en especies que presentan una corteza con circunvoluciones, como el gato, perro, mono y hombre; en aquellas especies con una corteza lisa, como la rata, el ratón y el conejo, el caudado recibe el nombre de estriado o caudado-putamen).

6. Altas concentraciones de $\mathrm{KCl}$ producen una despolarización aguda de las membranas neuronales, seguida de una ráfaga de potenciales de acción, que a su vez inducen una salida masiva de potasio, induciéndose una despolarización sostenida que se propaga a distancia. El resultado final es que los grupos neuronales afectados quedan incapacitados para funcionar durante un tiempo considerable (en nuestras condiciones experimentales, la inactivación dura alrededor de 3 horas). 
amnesia; los de entrenamiento intermedio, una amnesia moderada, mientras que la memoria de los que fueron sobre-entrenados no difirió de la de los grupos controles.

Esta serie experimental demostró, claramente, que el efecto protector del aprendizaje incrementado también sucede cuando éste es mediado por reforzadores positivos; en su conjunto, los datos presentados también demuestran que dicho efecto no es privativo de una sola especie animal, sino que cuando menos se manifiesta en roedores y felinos. También es importante hacer notar que el alto nivel de entrenamiento protege a la memoria en contra de los efectos de un buen número de agentes amnésicos, tales como las lesiones permanentes, la inactivación reversible producida por la lidocaína, la tetrodotoxina y el $\mathrm{KCL}$, bloqueadores de receptores a neurotransmisores como la atropina, la escopolamina, la bicuculina y la picrotoxina, así como agentes que depletan la serotonina cerebral.

La información descrita en este artículo dio lugar a la proposición de dos modelos teóricos que permiten dar una interpretación sencilla de los datos. Estos son los denominados modelos en serie y paralelo de la memoria (Prado-Alcalá, 1995), que explicaremos someramente a continuación.

En los casos que hemos referido, y en muchos más, si se interfiere con la actividad normal de una estructura cerebral determinada se produce una deficiencia en la consolidación o en la retención de la memoria. Lo mismo ocurre si se interfiere con la actividad de alguna otra estructura, y así sucesivamente. El punto es que existe un conjunto de núcleos cerebrales que son indispensables para que se establezca la memoria, y que basta con que uno sólo de ellos no funcione correctamente para que la información derivada del aprendizaje no sea almacenada. Estos hechos nos permitieron postular que estas estructuras están conectadas funcionalmente, en serie, y que la información derivada de una experiencia de aprendizaje debe transitar por todas ellas para llegar a algún centro de integración, de cuya activación depende la consolidación de la memoria.

El segundo modelo postula que en condiciones de aprendizaje mediado por altos niveles de reforzadores positivos o negativos, o por un número incrementado de ensayos o de sesiones de entrenamiento (o por alguna combinación de estos factores), las estructuras que originalmente estaban conectadas en serie (u otras estructuras sumadas a las originales), ahora sufren un cambio, conectándose funcionalmente en paralelo. De esta forma, aunque alguna de las estructuras del circuito no funcione normalmente o esté dañada, la actividad derivada de la experiencia de aprendizaje podrá seguir su trayecto hacia el posible centro integrador, haciendo posible la consolidación de la memoria. Las representaciones esquemáticas de ambos modelos se pueden ver el las Figuras 1 y 2. 

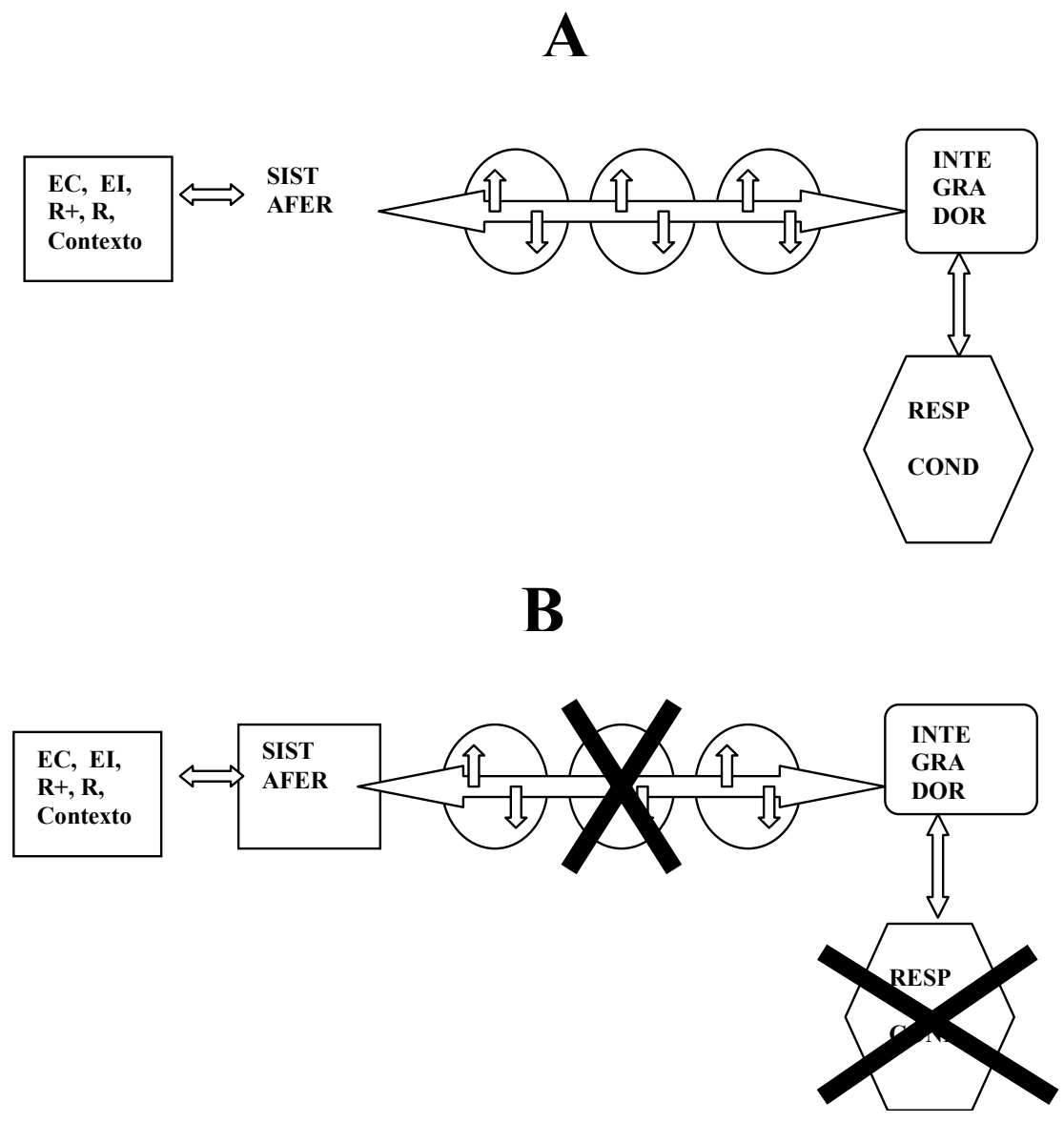

Figura 1. Modelo que representa la manera en que en condiciones de aprendizaje considerado normal, la interferencia con la actividad de estructuras cerebrales produce amnesia. La información de la situación de aprendizaje es captada por los sistemas sensoriales o aferentes (SIST AFER) y es relevada a las distintas estructuras (óvalos) que intervienen en el proceso de consolidación de la memoria; a su vez, estas se comunican con un Integrador de información que permite el almacenamiento o consolidación, así como la ejecución de la respuesta condicionada (RESP COND). En este sistema, el flujo de información es bidireccional entre todos los elementos, que están conectados, funcionalmente, en serie. Ver detalles en el texto. 


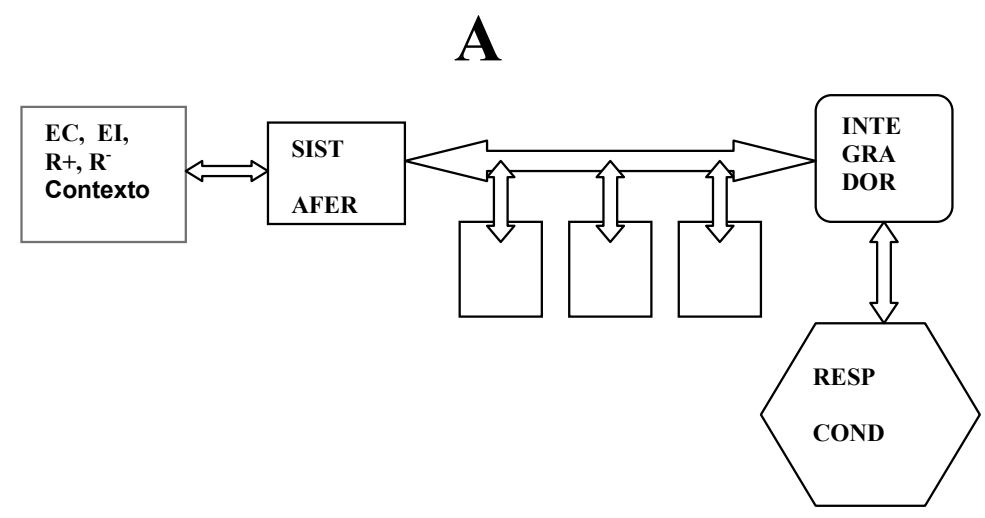

B

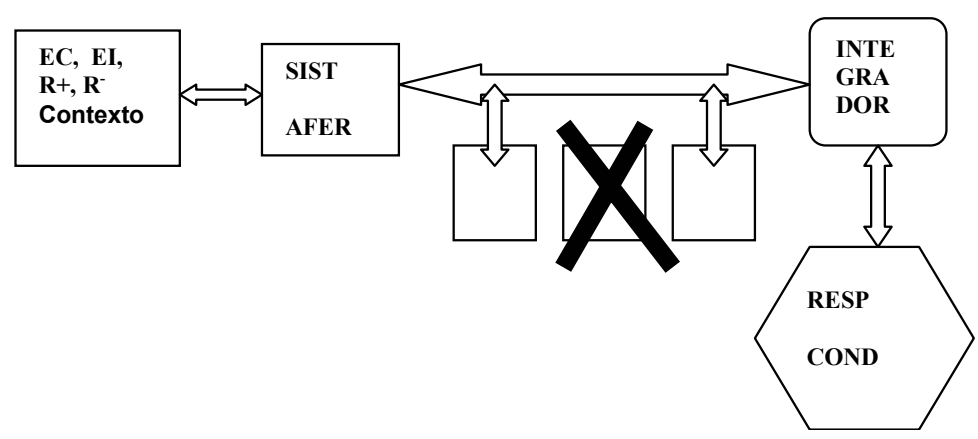

Figura 2. Modelo que representa la manera en que el aprendizaje incrementado protege en contra de la amnesia que habitualmente se produce por la aplicación de tratamientos que típicamente impiden la consolidación de la memoria, interfiriendo con la actividad de ciertas estructuras cerebrales. La información procedente de la situación de aprendizaje es captada por los sistemas sensoriales o aferentes (SIST AFER) y es relevada a las distintas estructuras que intervienen en el proceso de consolidación de la memoria (rectángulos entre SIST AFER e INTEGRADOR); a su vez, estas se comunican con un Integrador de información que permite el almacenamiento o consolidación, así como la ejecución de la respuesta condicionada (RESP COND). En este sistema, el flujo de información es bidireccional entre todos los elementos, pero como consecuencia del aprendizaje incrementado, las estructuras involucradas en la consolidación de la memoria sufren un cambio funcional y se reconectan en paralelo. Ver detalles en el texto. 


\section{REFERENCIAS BIBLIOGRÁFICAS}

Altman, H. J., \& Normile, H. J. (1986). Enhancement of the memory of a previously learned aversive habit following pre-test administration of a variety of serotonergic antagonists in mice. Psychopharmacology, 90, 24-27.

Ambrogi Lorenzini, C. G., Baldi, E., Bucherelli, C., Sacchetti, B., \& Tassoni G. (2000). Neural topography and chronology of memory consolidation: a review of functional inactivation findings. Neurobiology of Learning and Memory, 73, 282-283.

Ambrogi-Lorenzini, C. G., Baldi, E., Bucherelli, C., \& Tassoni G. (1994). Passive avoidance response disruption by post-training substantia nigra functional tetrodotoxin inactivation in the rat. Archives Italiennes de Biologie, 132, 85-92.

Archer, T. (1982). "Serotonin and fear retention in the rat. Journal of Comparative and Physiological Psychology, 96, 491-516.

Bailey, C. H., Bartsch, D., \& Kandel, E. R. (1996). Toward a molecular definition of long-term memory storage._Proceedings of the National Academy of Sciences of the United States of America, 93, 13445-13452.

Bammer, G. (1982). Pharmacological investigations of neurotransmitter involvement in passive avoidance responding: a review and some new results. Neuroscience and Biobehavioral Reviews, 6, 47-296.

Bartus, R. T., Dean, R. L., Pontecorvo, M. J., \& Flicker, C. (1985). The cholinergic hypothesis: a historical overview, current perspective, and future directions. Annals of the New York Academy of Sciences, 444, 332-358.

Bermúdez-Rattoni, F., \& Prado-Alcalá, R. A. (2001). Memoria. ¿En dónde está y cómo se forma? México: Editorial Trillas.

Cobos-Zapiaín, G. G., Salado-Castillo, R., Sánchez-Alavez, M., Quirarte, G.L., RoldánRoldán, G., \& Prado-Alcalá, R. A. (1996). High level of footshock during inhibitory avoidance training prevents amnesia induced by intranigral GABA antagonists. Neurobiology of Learning and Memory, 65,202-206.

Cruz-Morales, S. E., Durán Arévalo, M., Díaz del Guante, M. A., Quirarte, G., \& PradoAlcalá, R. A. (1992). A threshold for the protective effect of over-reinforced passive avoidance against scopolamine-induced amnesia. Behavioral and Neural Biology, 57, 256-259.

Da Cunha, C., Angelucci, M. E. M., Canteras, N. S., Wonnacott, S., \& Takahashi, R. N. (2002). The lesion of the rat substantia nigra pars compacta dopaminergic neurons as a model for Parkinson's disease memory disabilities. Cellular and Molecular Neurobiology, 22, 227-237.

Da Cunha, C., Wietzikoski, S., Wietzikoski, E. C., Miyoshi, E., Ferro, M. M., AnselmoFranci, J. A., \& Canteras, N. S.et al. (2003). Evidence for the substantia nigra pars compacta as an essential component of a memory system independent of the hippocampal memory system Neurobiology of Learning and Memory, 79, 236-242.

Decker, M. W., \& McGaugh, J. L. (1991). The role of interactions between the cholinergic system and other neuromodulatory systems in learning and memory. Synapse, 7, 151-168.

Díaz del Guante, M.A., Rivas-Arancibia, S., Quirarte, G., \& Prado-Alcalá, R. A. (1990). Over-reinforcement protects against memory deficits induced by muscarinic 
blockade of the striatum. Boletín de Estudios Médicos y Biológicos, México, 38, 49-53.

Díaz del Guante, M. A., Rivas, M., Prado-Alcalá, R. A., \& Quirarte, G. L. (2004). Amnesia produced by pre-training infusion of serotonin into the substantia nigra. Neuroreport, 15, 2527-2529.

Durán-Arévalo, M., Cruz-Morales, S. E., \& Prado-Alcalá, R. A. (1990). Is acetylcholine involved in memory consolidation of over-reinforced learning? Brain Research Bulletin, 24, 725-727.

Fibiger, H. C. (1991). Cholinergic mechanisms in learning, memory and dementia: a review of recent evidence. Trends in Neurosciences, 14, 220-223.

Giordano, M. \& Prado-Alcalá, R. A. (1986). Retrograde amnesia induced by post-trial injection of atropine into the caudate-putamen. Protective effect of the negative reinforcer. Pharmacology, Biochemistry, and Behavior, 24, 905-909.

Gold, P. E. (2003). Acetylcholine modulation of neural systems involved in learning and memory. Neurobiology of Learning and Memory, 80, 194-210.

Izquierdo, I., \& Medina, J. H. (1993). Role of the amygdala, hippocampus and entorhinal cortex in memory consolidation and expression. Brazilian Journal of Medical and Biological Research, 26, 573-589.

Martínez, I., Quirarte, G. L., Díaz-Cintra, S., Quiroz, C., \& Prado-Alcalá, R.A. (2002), Effects of lesions of hippocampal fields CA1 and CA3 on acquisition of inhibitory avoidance. Neuropsychobiology, 46, 97-103.

McDonald, R. J., \& White, N. M. (1993). A triple dissociation of memory systems: Hippocampus, amygdala, and dorsal striatum. Behavioral Neuroscience, 107: 3-22.

McGaugh, J. L. (2000). Memory - a century of consolidation. Science, 287, 248-251.

McGaugh, J. L.(2002). Memory consolidation and the amygdala: a systems perspective. Trends in Neurosciences, 25, 456.

McGaugh, J. L., McIntyre, C. K., \& Power, A. E. (2002). Amygdala modulation of memory consolidation: interaction with other brain systems. Neurobiology of Learning and Memory, 78, 539-552.

Meneses, A. (2003). A pharmacological analysis of an associative learning task: 5$\mathrm{HT}(1)$ to $5-\mathrm{HT}(7)$ receptor subtypes function on a pavlovian/instrumental autoshaped memory. Learn Mem. 2003 Sep-Oct;10(5):363-372.

Meneses, A. (1999). 5-HT system and cognition. Neuroscience and Biobehavioral Reviews, 23, 1111-1125.

Meneses, A, (2004). Manuel-Apolinar L, Rocha L, Castillo E, Castillo C. Expression of the 5 -HT receptors in rat brain during memory consolidation. Behavioural Brain Research, 152, 425-36

Müller, G. E., \& Pilzecker, A. (1900). Experimentelle beitrage zur lehre vom gedachtnis. Zeitschrift fur Psychologie,_Supplementum, 1-288.

Ögren, S. O. (1985). Evidence for a role of brain serotonergic neurotransmission in avoidance learning. Acta Physiologica Scandinavica. Supplementum, 544, 1-71.

Ögren, S. O. (1986a). Analysis of the avoidance learning dificit indiced by the serotonin releasing compound p-chloroamphetamine. Brain Research Bulletin, 16, 645-660.

Ogren, S. O. (1986b). Serotonin receptor involvement in the avoidance learning deficit caused by pchloroamphetamine-induced serotonin release. Acta Physiologica Scandinavica, 126, 449-462 
Packard, M. G., \& Knowlton, B. J. (2002). Learning and memory functions of the Basal Ganglia. Annual Review of Neuroscience, 25, 563-1293.

Parent, M.B., \& McGaugh, J. L. (1994). Posttraining infusion of lidocaine into the amygdala basolateral complex impairs retention of inhibitory avoidance training. Brain Research, 661, 97-103.

Parent, M. B., Quirarte, G. L., Cahill, L., \& McGaugh, J. L. (1995). Spared retention of inhibitory avoidance learning after posttraining amygdala lesions. Behavioral Neuroscience, 109, 803-807.

Parent, M.B., Tomaz, C., \& McGaugh, J. L. (1992). Increased training in an aversively motivated task attenuates the memory-impairing effects of posttraining $\mathrm{N}$-methylD-aspartate-induced amygdala lesions. Behavioral Neuroscience, 106, 789-797.

Parent, M. B., West, M., \& McGaugh, J. L. (1994). Memory of rats with amygdala lesions induced 30 days after footshock-motivated escape training reflects degree of original training. Behavioral Neuroscience, 108, 1080-1087.

Pérez-Ruiz, C., \& Prado-Alcalá, R. A. (1989). Retrograde amnesia induced by lidocaine injection into the striatum: Protective effect of the negative reinforcer. Brain Research Bulletin, 22, 599-603.

Power, A. E. (2004). Muscarinic cholinergic contribution to memory consolidation: with attention to involvement of the basolateral amygdala. Current Medicinal Chemistry, 11, 987-996.

Prado-Alcalá, R. A. (1985). Is cholinergic activity of the caudate nucleus involved in memory? Life Sciences, 37, 2135-2142.

Prado-Alcalá, R. A. (1995). Serial and parallel processing during memory consolidation. In J. L. McGaugh, F. Bermúdez-Rattoni \& R. A. Prado-Alcalá (Eds.), Plasticity in the Central Nervous System. Learning end Memory. (pp. 57-65). New Jersey: Lawrence Erlbaum Publishers,

Prado-Alcalá, R. A., Bermúdez-Rattoni, F., Velázquez-Martínez, D., \& Bacha, M.G. (1978). Cholinergic blockade of the caudate nucleus and spatial alternation performance in rats: overtraining-induced protection against behavioral deficits. Life Sciences, 23, 889-896.

Prado-Alcalá, R. A., \& Cobos-Zapiaín, G. G. (1979). Interference with caudate nucleus activity by potassium chloride. Evidence for a "moving" engram. Brain Research, 172, 577-583.

Prado-Alcalá, R. A., \& Cobos-Zapiaín, G. G. (1977). Learning deficits induced by cholinergic blockade of the caudate nucleus as a function of experience. Brain Research, 138, 190-196.

Prado-Alcalá, R. A., Grinberg, Z. J., Alvarez-Leefmans, F. J., Gomez, A., Singer, S. \& Brust-Carmona, H. (1972). A possible caudate-cholinergic mechanism in two instrumental conditioned responses. Psychopharmacologia (Berl.), 25, 339-346.

Prado-Alcalá, R. A., Kaufman, P. \& Moscona, R. (1980). Scopolamine and KCL injections into the caudate-putamen. Overtraining- induced protection against deficits of learning. Pharmacology, Biochemistry, and Behavior, 12, 249-253.

Prado-Alcalá, R. A., Quiroz, C. R., Garín, M. E., Díaz Trujillo, A., Díaz del Guante, M. A., Galindo, L. E., Martínez, I., \& Quirarte, G. L. (2004). Memoria: consolidación y experiencia. In: J. Velásquez Moctezuma (Ed.), Temas Selectos de Neurociencias III, (pp. 121-138). México: Universidad Autónoma Metropolitana. 
Quirarte, G. L., Cruz-Morales, S. E., Díaz del Guante, M. A., García, M., \& Prado-Alcalá, R.A. (1993). Protective effect of under-reinforcement of passive avoidance against scopolamine-induced amnesia. Brain Research Bulletin, 32, 521-524.

Quiroz, C., Martínez, I., Quirarte, G. L., Morales, T., Díaz-Cintra, S., \& Prado-Alcalá, R.A. (2003). Enhanced inhibitory avoidance learning prevents the memory-impairing effects of posttraining hippocampal inactivation. Experimental Brain Research, 221, 400-402.

Rodrigues, S. M., Schafe, G. E., \& LeDoux, J. E. (2004). Molecular mechanisms underlying emotional learning and memory in the lateral amygdala. Neuron, 44, 7591.

Routtenberg, A., \& Holzman, N. (1973). Memory disruption by electrical stimulation of substantia nigra, pars compacta. Science, 181, 83-96.

Salado-Castillo, R., Sánchez-Alavez, M., Quirarte, G. L., \& Prado-Alcalá, R. A. (1995). Reversible lesions of striatum, amygdala, and substantia nigra after inhibitory avoidance: Differential effects of high lad low footshock intensity. $25^{\text {th }}$ Annual Meeting, Society for Neuroscience, San Diego, California, U.S.A.

Solana-Figueroa, R., Quirarte, G. L., \& Prado-Alcalá, R. A. (1999). Effects of pre-training systemic administration of $\mathrm{p}$-chloroamphetamine on inhibitory avoidance trained with high and low foot-shock. Revista Mexicana de Psicología, 2, 211-215.

Solana-Figueroa, R., Salado-Castillo, R., Quirarte, G. L., Galindo, L. E., \& Prado-Alcalá, R. A. (2002). Enhanced inhibitory avoidance training protects against the amnesic effect of p-chloroamphetamine. Life Sciences, 71, 391-399.

Stork, O., \& Welzl, H. (1999). Memory formation and the regulation of gene expression. Cellular and Molecular Life Sciences, 55, 575-592. 